\title{
Bibliometric study of the reverse salient concept
}

\author{
Ozgur Dedehayir \\ Tampere University of Technology (FINLAND) \\ ozgur.dedehayin@tut.fi
}

Received September 2009

Accepted December 2009

\begin{abstract}
In the development of technological systems the focus of system analysis is often on the sub-system that delivers insufficient performance - i.e. the reverse salient which subsequently limits the performance of the system in its entirety. The reverse salient is therefore a useful concept in the study of technological systems and while the literature holds numerous accounts of its use, it is not known how often, in which streams of literature, and in what type of application the concept has been utilized by scholars since its introduction by Thomas Hughes in 1983. In this paper we employ bibliometric citation analysis between 1983 and 2008, inclusively, to study the impact of the reverse salient concept in the literature at large as well as study the dissemination of the concept into different fields of research. The study results show continuously growing number of concept citations in the literature over time as well as increasing concept diffusion into different research areas. The analysis of article contents additionally suggests the opportunity for scholars to engage in deeper conceptual application. Finally, the continuing increase in the number of citations highlights the importance of the reverse salient concept to scholars and practitioners.
\end{abstract}

Keywords: technological system, reverse salient, bibliometric study, citation life cycle

\section{Introduction}

Studying technology through a systemic view offers scholars unique advantages that are inaccessible in studying technology as a unitary phenomenon. The 
perspective of technology as a hierarchically structured entity that comprises different levels of interconnected systems and sub-systems (Hughes, 1983; Murmann \& Frenken, 2006) enables scientists and practitioners to reveal the interaction of sub-systems within the collective system. Consequently, the researcher increases his/her understanding of the technology as well as the technology's change over time.

One profound characteristic that emerges from the systemic study of technologies is the state of inequality that exists at any point in time between different technological sub-systems, brought about by the complexity of the system as well as its dynamic nature. Subsequently, the performance level of a particular subsystem is inevitably lower than the levels reached by other sub-systems. This state of performance unevenness carries significance in scientific analyses of technological systems because the loci of interest are often the factors which, due to their own limitations, constrain collective system development. Scientific literature studying technological systems has therefore utilized different concepts which describe this phenomenon, albeit with slight nuances; including bottleneck and technological disequilibrium. One such concept, the reverse salient, is introduced by Thomas Hughes in his 1983 seminal work, 'Networks of Power: Electrification in Western Society, 1880-1930', referring to the under-performing systemic member, which acts as a retardant and hampers the development of the overall system performance. Due to conceptual difference with respect to other concepts, the reverse salient has received attention from scholars who have appropriated its use in the study of technological systems in certain context. While the literature holds numerous accounts of its use, the importance of the concept to the scientific community is unknown. Moreover, it is not known how often, in which streams of literature, and in what type of application the concept has been utilized by scholars since its introduction.

The purpose of this paper is to study the literature in order to gain greater insight into the use of the reverse salient concept, first introduced by Thomas Hughes in 1983. For this purpose we firstly perform bibliometric citation analysis to observe the temporal change in the number of citations of the reverse salient concept in the literature at large. In turn, we assess the dissemination of the concept by first analyzing the number of different journals that cite the concept, and second, analyzing the number of different contexts of study that cite the concept, over time. In our analyses we consider the life cycle of concept citations as an indicator 
of the concept's importance to the literature and subsequently assess the significance of the reverse salient concept in the study of technological systems. Finally, we analyze the content of the articles citing the reverse salient concept and determine the depth of conceptual application employed by the authors.

The structure of the paper is as follows. We begin by providing a theoretical as well as an historical perspective of the concept together with accounts of its application in various contexts and research streams. In the following section, the paper formulates the employed methodology, comprising a bibliometric study that covers 97 journal articles collected from different electronic citation databases. The study results offer the temporal change in the number of concept citations in the literature at large, then more specifically in different journals and different contexts of study, and finally with respect to depth of conceptual use, between the years 1983 and 2008, inclusively. These results are subsequently evaluated to identify potential expansion of the scope of research in which the reverse salient concept can be applied.

\section{Theoretical background}

\subsection{The technological system}

In contrast to studying technology as a unitary phenomenon, scholars have recognized certain advantages in taking a systemic view of technology. As a system, technology has been analyzed as a hierarchically structured entity that comprises different levels of interconnected systems and sub-systems (see for example: Henderson \& Clark, 1990; Murmann \& Frenken, 2006; Rosenberg, 1976; Rosenkopf \& Nerkar, 1999) and as an actor network that constitutes different although interrelated components (see for example: Callon, 1987). Most importantly, these perspectives advocate a meta-level view and analysis of technology, through which the interaction of sub-systems or actors is brought forth. The result is the increase in explanatory power of the scholar's analysis of technology and its evolution.

The adoption of a systemic perspective in the study of technological evolution, as also demonstrated in biological, socio-cultural, and organizational studies of evolution in earlier research, enhances understanding of the evolutionary mechanisms which are at work both within hierarchical levels as well as across 
hierarchical levels (Rosenkopf \& Nerkar, 1999). This means that individual subsystems do not merely evolve, but rather, they co-evolve interdependently with other sub-systems. Furthermore, the systemic view recognizes evolutionary trajectories of technology which are simultaneously observed at every level of the system hierarchy subsequent to variation, selection, and retention processes (Rosenkopf \& Nerkar, 1999). As a result it is possible to identify the location of a punctuated, discontinuous development at, say, a lower-order sub-system, which may translate into incremental development at a higher-order level of the system echelon. Therefore, the systemic view could go some way into opening the black box of technology, thereby reducing the ambiguity in the nature of technological change by considering the hierarchical structure and complexity of technological systems.

On the one hand technological systems have been treated as a make-up of purely technical sub-systems. In this manner, an aircraft can be considered as a system that has some major technical sub-systems including the engine, which can be decomposed into smaller technical sub-systems, such as the compressor, combustion system, and turbine, which are subsequently divisible yet again into smaller parts (Constant, 1987; Murmann \& Frenken, 2006). Similarly, the study of complex product systems (CoPS) (see for example: Hobday, 1998; Prencipe, 2000) focuses on the technical components (high cost products, systems, and networks) which constitute technological systems. A technical emphasis allows scholars to study the system as an entire whole by analyzing the relationship and interaction between its technical sub-systems, for example in studying industrial change subsequent to innovations within the technological system (Henderson \& Clark, 1990) or in studying the historical developments of technological systems (Rosenberg, 1976). Hence, social factors such as the firms which produce the technical sub-systems are seen as external to the system.

Alternatively, technological systems have been treated as socio-technical systems that include social components in addition to the technical. The socio-technical perspective allows the analysis of technological systems in the context of not only the technical artifacts but also the end-users of these artifacts as well as the makers of the artifacts. This view has been greatly advocated by the social constructivist approach to understanding technology systems (Bijker, Hughes, \& Pinch, 1987), although it does not necessarily belong only to this area of investigation. The socio-technical perspective is clearly advantageous when 
scholars wish to emphasize the impact of social groups on the technology system and its change over time. One of the most influential works that illustrates the socio-technical approach is Thomas Hughes' Networks of Power: Electrification in Western Society, 1880-1930. In his seminal work, Hughes (1983) traces the development of the electric technology system from its birth until the early periods of the twentieth century. He assumes a socio-technical standpoint in his book by alluding at the very outset that throughout its progress, the electric system had not only inflicted change upon its social environment but had also been altered by it.

\subsection{The socio-technical system and its evolution}

As part of his systemic framework, where change causality is sourced bilaterally from technical and social factors, Hughes (1983) provides at least two mechanisms for systemic evolution. The first mechanism is the interaction of the technological system with its environment, which consists of factors not in the system's control and thus present uncertainties. This is exemplified by the dependence of the electric system on an environmental factor such as the supply of fossil fuels. Technological systems thus carry incentives to evolve by internalizing their environments over time and so minimize potentially harmful ramification of uncertainties. A second explanation of technological system evolution is the system's endeavor to solve problems or fulfill goals (Hughes, 1987).

Hughes proposes that technological systems pass through particular phases during the system's evolution. The first of these phases sees the invention and development of the system, owed greatly to the efforts of inventors and entrepreneurs. Considering the initial phase of the electric technological system, for example, Hughes highlights the imperative role played by individuals such as Thomas Edison. The second stage is the era of technological transfer from one region or society to others. Hughes exemplifies such technological transfer through the dissemination of Edison's electric system from New York City to other cities such as London and Berlin. The third phase of systemic evolution is marked by a period of system growth. This is the time of expansion when the technological system strives to improve its performance, for instance with respect to economic outcomes or output efficiency. Hughes explicates that in this developmental phase, technological systems are dependent on the satisfactory evolution of all system components' performance. Within the hierarchically nestled system, the development of technological systems is therefore reliant on the reciprocated and 
interdependent cause and effect processes amongst social and technical components. More accurately, the modus operandi of such developmental change may be described as co-evolutionary, where the balanced co-evolution of system components carries significance in establishing desired system progress (Hughes, 1983). In other words, components of the system which evolve at a sufficient pace contribute positively to the collective development. Conversely, a sub-system which does not develop sufficiently prevents the technology system achieving its targeted development. Hughes (1983) names these problematic sub-systems reverse salients.

A reverse salient is the inverse of a salient that depicts the forward protrusion along an object's profile or a line of battle (Hughes, 1987). Hence, reverse salients are the backward projections along similar, continuous lines. With respect to technological system development, reverse salients refer to the components of that system which have strayed behind the advancing performance frontier of the system (Hughes, 1983). They are therefore the underperforming components which hamper the progress or which prevent the fulfillment of potential development of the collective system. In line with the socio-technical standpoint, reverse salients can be technical elements such as motors and capacitors of an electric system, or social elements such as organizations or productive units.

Because reverse salients establish the limited pace of system development, it is imperative that they are corrected, where correction is attained through incremental or radical inventions (Hughes, 1987). Consequently, the reverse salient forms a nexus or focusing device (Rosenberg, 1969) for technological system stakeholders, in particular innovators and organizations, which congregate around the retardant and strive to remove it through innovation processes. If the reverse salient is not able to be corrected within the bounds of the existing technological system through incremental innovations, then only radical innovations can successfully resolve the problematic sub-system. The outcome of radical innovations is the creation of a new and different technological system, as witnessed through the innovation of the alternating-current system which overcame the low cost distribution hurdle of the electric technological system, where the direct-current system could not (Hughes, 1983). 


\subsection{The reverse salient concept}

The reverse salient is a useful concept for both scholars and practitioners in analyzing technological system evolution. This is because quite often the loci of interest in scientific analyses of technological systems are the factors which limit system development. Furthermore, these limiting factors may be more than merely technical components, and at times, quite importantly, may be social components as well. In this way the conceptualization of system performance hindering factors as reverse salients can be more applicable in certain contexts in contrast to similar or overlapping concepts such as bottleneck and technological imbalance (Rosenberg, 1976).

Although the reverse salient and bottleneck can be used interchangeably in particular contexts (see for example: Fransman, 2001; Geels, 2006; Geyer \& Davies, 2000; Markard \& Truffer, 2006) often the reverse salient refers to the subsystem that not only curbs the performance or output of the collective system but also requires correction because of its limiting affect. This is not necessarily the case with bottlenecks. For instance, a particular system's output performance may be compromised due to a bottleneck sub-system but the bottleneck will not require improvement if the overall system's current output performance is satisfactory. If, on the other hand, higher performance would be required of the same system, the bottleneck may emerge as a reverse salient that holds the system back from attaining that higher output performance.

A similar overlap exists between the reverse salient and technological imbalance concepts (see for example: den Hond, 1998; Fransman, 2001; Takeishi \& Lee, 2005). Rosenberg's (1976) imbalance arises from the comparison of technological components of complex machines or operations, which reveal that at any point in time machine component parts will vary in their ability to exceed their existing performance levels, attributable to some limiting component. This condition results in the disequilibrium of technologies within the same system. Rosenberg illustrates this disequilibrium effect through the example of a machining operation in the manufacturing of bicycle hubs. In this example, despite performance improvements which quicken the sub-process of hub forming, the overall pace of hub production remains unchanged. Benefits from the forming process improvement are not realized until the interdependent drilling sub-process quickens its speed of production as well. A condition of disequilibrium therefore emerges between two 
processes, embedded within the same complex operation. Hence, the technological imbalance described by Rosenberg can equally be seen as a state of reverse salience in a technological system when studying only its technical components. However, the reverse salient concept differs in that it can be used to study imbalances not only between different technical components, but also social components.

Historical developments in a variety of technological systems have been used in the literature to illustrate reverse salience. In his seminal work, Hughes (1983) gives account of the development of Edison's direct-current electric system towards the objective of supplying electricity within a defined region of distribution. Subsystems such as the direct-current generator were identified as reverse salients and corrected. However, perhaps the most notable limitation of the direct-current system was its low voltage transmission distance. The reverse salience in this case was the cost of distributing electricity beyond a certain range. To reduce costs, Edison introduced a three-wire system to replace the previously installed two-wire alternative and trialed different configuration of generators, as well as the usage of storage batteries. While these had a positive impact, they did not correct the reverse salient completely. The satisfactory resolution of the problem of costly transmission and distribution of low voltage electricity was eventually provided by the radical innovation of the alternating-current system (Hughes, 1983).

Numerous authors have since offered their accounts based on the analysis of different technological systems. MacKenzie (1987) has pinpointed the gyroscope sub-system as a technical reverse salient in the ballistic missile technological development, where the systemic objective has been to increase missile accuracy. With the objective of proliferating mobile music throughout the end-user market, Takeishi and Lee (2005) have argued that music copyright managing institutions have acted as a social reverse salient in the evolution of the mobile music technology system in Japan and Korea. According to Mulder and Knot (2001), the development of the PVC (polyvinyl chloride) plastic technology system has been sequentially hampered by several states of reverse salience, including: difficulty to process PVC material, quality of manufactured products, health concerns for individuals exposed to effluent from PVC manufacturing facilities, and finally the carcinogenetic nature of vinyl chloride. 


\section{Data and methods}

\subsection{The reverse salient concept in the literature}

The reverse salient has emerged as a useful concept since its first introduction in the literature in 1983. Notwithstanding, it is not known how often, in which streams of literature, and in what type of application the concept has been utilized by scholars since its introduction. To understand the value of the concept as seen by scholars and at the same time predict future avenues of conceptual use it is necessary to investigate the concept's utilization in the literature thus far. This understanding is important for both scholars and practitioners because their analysis of technological systems requires the selection of conceptual tools, such as the reverse salient, whereby the value afforded by previous scholars on the conceptual tool establishes its potential selection among other concepts.

On the premise that the number of citations of a concept indicates the impact of that concept on the literature, we derive several propositions as to the expected adoption of the reverse salient concept in the literature from the time of its inception. Here, we treat a concept as an intellectual idea or information which experiences similar dissemination pattern within scientific fields as the adoption of innovations (Ley, 2003). Using the number of citations as a proxy for concept dissemination, we predict the emergence of an S-curve pattern (Cano \& Lind, 1991; Lariviere, Archambault, \& Gingras, 2007; Ley, 2003; Schwartz \& Ibaraki, 2001; Schwartz \& Fang, 2007) that is marked by rapid increase in the cumulative number of citations early on, followed by a diminishing rate of increase as the concept's value in the eyes of scholars reduces and the concept nears obsolescence. As the results of Cano and Lind's (1991) citation life cycle analysis for seminal works indicate, the temporal change in the number of citations may follow either a sharp rise early after concept introduction (i.e. in the first four to seven years) and gradually reduce thereafter, or increase gradually for some period after initial introduction (i.e. for the first six years) and then begin a period of take-off.

We firstly focus on the citations of the reverse salient in the literature at large. After its introduction in 1983, we expect some time to elapse before scholars become aware of the concept and its potential applicability. Cano and Lind (1991) have for example identified a three year lapse from the time of a work's 
introduction and its first citation. In time, however, the number of adopters is expected to increase as the growing number of conceptual applications in the literature provides greater visibility and illustrate the concept's application potential and importance. This leads us to derive the following proposition:

- Proposition 1: The number of citations of the reverse salient concept will increase in time after its introduction.

The reverse salient concept is widely applicable in different contexts despite originating in the study of the electric technological system analyzed by Hughes. Hence, we anticipate that the concept is likely to disseminate into different researched fields and appear in different publication outlets (in this article we focus on journals as publication outlets). Moreover, the pattern of concept diffusion is expected to follow an S-curve (see for example: Hargens, 2000), although the due to the relatively recent introduction of the concept, it is uncertain whether sufficient time has elapsed for the manifestation of the complete S-curve. Nevertheless, we predict that the concept will initially receive interest from only limited fields of research as awareness among the scientific community remains limited. Over time however, we believe that the concept will receive attention from a growing number of both researched fields (i.e. technological systems that have been analyzed) and journals. This leads us to state the following propositions:

- Proposition 2: The number of journals citing the reverse salient concept will increase in time after its introduction.

- Proposition 3: The number of research fields citing the reverse salient concept will increase in time after its introduction.

In addition to quantitative indicators, the mode of application of the reverse salient concept is also interesting to study. This qualitative aspect informs of the depth of conceptual engagement the authors make as they analyze a particular technological system. We estimate a change in the degree of conceptual application to take place over time, as scholars become more familiar with the concept and increase their knowledge. Therefore, soon after the concept's introduction in 1983, we expect awareness and knowledge to remain limited, consequently resulting in shallow conceptual utilization. In other words, in this early phase, scientific work that makes use of the reverse salient concept will remain shy of comprehensive application of the concept in the analysis of 
technological systems. However, in time, we expect conceptual understanding to increase, especially with growing visibility, leading to greater depth of conceptual usage and in turn further development of the concept. This argument leads to the following proposition:

- Proposition 4: The number of shallow conceptual applications will reduce, while the number of deep conceptual applications will increase over time.

\subsection{The data}

The objective of this paper is to investigate the impact of the reverse salient concept on the general body of literature - in other words its utilization by scholars - since its introduction by Hughes in 1983. The research method employed here is firstly a bibliographic citation analysis, which describes the impact of one research on another (Brown \& Gardner, 1985). Moreover, citations of a particular concept indicate its innovativeness or usefulness as scholars consider them to be worthy of reference and application in their own research. Citation analysis is also advantageous because of its objective nature thus rendering it free of perceptions of the researcher, contrary to other forms of similar research (e.g. opinion surveys) (Chandy \& Williams, 1994).

In this study we considered only journal articles as the outlet for scholarly work, based on the argument that articles are more competitive than other modes of communication in scholarly discipline (Chandy \& Williams, 1994) and therefore more comprehensive. We collected all journal articles citing the exact phrase "reverse salient", "reverse salients", and "reverse salience", between 1983 and 2008, inclusively. For this process we accessed journals that were listed on a number of databases: JSTOR, ScienceDirect, EBSCOhost, ProQuest, Sage Premier, ISI Web of Knowledge, IEEE Xplore, and SpringerLink.

Our initial search results included journal introductions, conclusions, editor comments, commentaries, responses to critique, and book reviews. We decided to filter these out of the scope of our analysis because our study focuses on the utilization of the reverse salient concept in the analysis of a technological system, and often the aforementioned works did not analyze a technological system. We also excluded articles which either falsely referred to the concept (e.g. "adverse salients" rather than "reverse salients") or where the search term (e.g. "reverse salient") appeared in the reference list but not at all in the main text of the article. 
Furthermore, we left out of our study any articles which were currently in press, as it remained equivocal as to when they would be published and hence, whether they would fall within the timeframe of our study. Finally, although we looked for citations from 1983 onwards, we could not access articles earlier than 1995 (nine articles in all) in the ScienceDirect database and were therefore forced to neglect these works.

All in all we acquired 97 journal articles to be included in our study and listed these in chronological order. By adopting a diachronous approach, thereby counting the number of citations made to a single concept in a longitudinal study (Cano \& Lind, 1991), we analyzed the temporal change in the number of reverse salient citations in the literature at large. It should be noted that in this longitudinal study, the data has not been corrected for population growth, for instance with respect to active researchers or the number of journals that may have increased over the years.

Next, to understand the adoption of the concept within different contexts, we collected the articles firstly in groups of the technological systems that had been studied (e.g. we collected all articles that had studied the aviation technological system into one group), and secondly in groups of the journals where the article had been published (e.g. we collected all articles that had appeared in the Research Policy journal into one group). We then analyzed the change in the number of different technological systems that had applied the reverse salient concept over time, and the change in the number of different journals that had cited the concept over time, respectively.

Finally, we analyzed the content of the articles (Kassarjian, 1977) in order to derive qualitative understanding of the reverse salient concept's utilization in the literature. Here, the collected articles were analyzed in the depth of conceptual application employed by the authors in their studies. We grouped articles into the shallow category if the articles had not integrated the reverse salient concept in a comprehensive manner. Typically, the articles which were allocated to this category had merely referred to the reverse salient concept in theory building or in the theoretical discussion of the article. Conceptual articles, which had subsequently not studied a technological system, were also allocated to the shallow category (while it can rightfully be argued that conceptual papers have significant depth of analysis, in this study we focused on the depth of conceptual use in the analysis of technological systems only, following the seminal work Hughes). On the contrary, if 
the authors had analyzed a specific technological system using the reverse salient concept we felt that they had engaged in deeper conceptual application. We classified these latter articles within the deep category. Typically, the articles which were judged to belong to this category had, as a minimum requirement, identified a reverse salient in the analyzed context (i.e. the technological system). Some of these works had further extended the discussion by considering the consequence and resolution of reverse salients. While such content analysis can be subjective, we tried to reduce the degree of subjectivity in our analysis by minimizing the number of categories into which the articles could be allocated with respect to the depth of conceptual application (only two categories were used). After this classification, we analyzed the change in the number of articles belonging to the shallow and deep categories over time, respectively.

\section{Results}

The temporal change in the number of citations of the reverse salient concept in the literature at large is shown in Figure 1. This graph indicates a rising number of citations per year from 1990 (the first journal article citing the concept) onwards. Hence, the cumulative number of citations shows an almost exponential growth for the timeframe of analysis, commensurate with our first proposition.

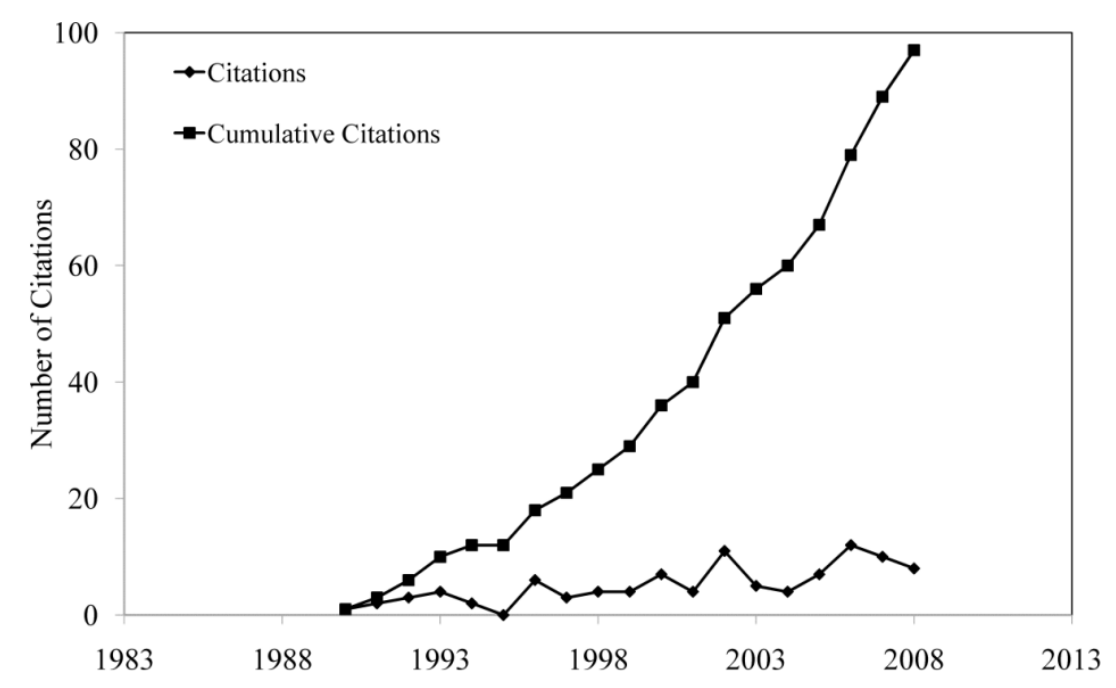

Figure 1. "Citations of the reverse salient concept in the literature at large".

The growing number of citations in the literature suggests that the reverse salient concept continues to hold relevance to the works of modern day scholars such that 
they feel it beneficial to use it in their work. Subsequently, the concept has been cited in a number of different periodicals. Figure 2 displays the journals that have cited the reverse salient two times or more.

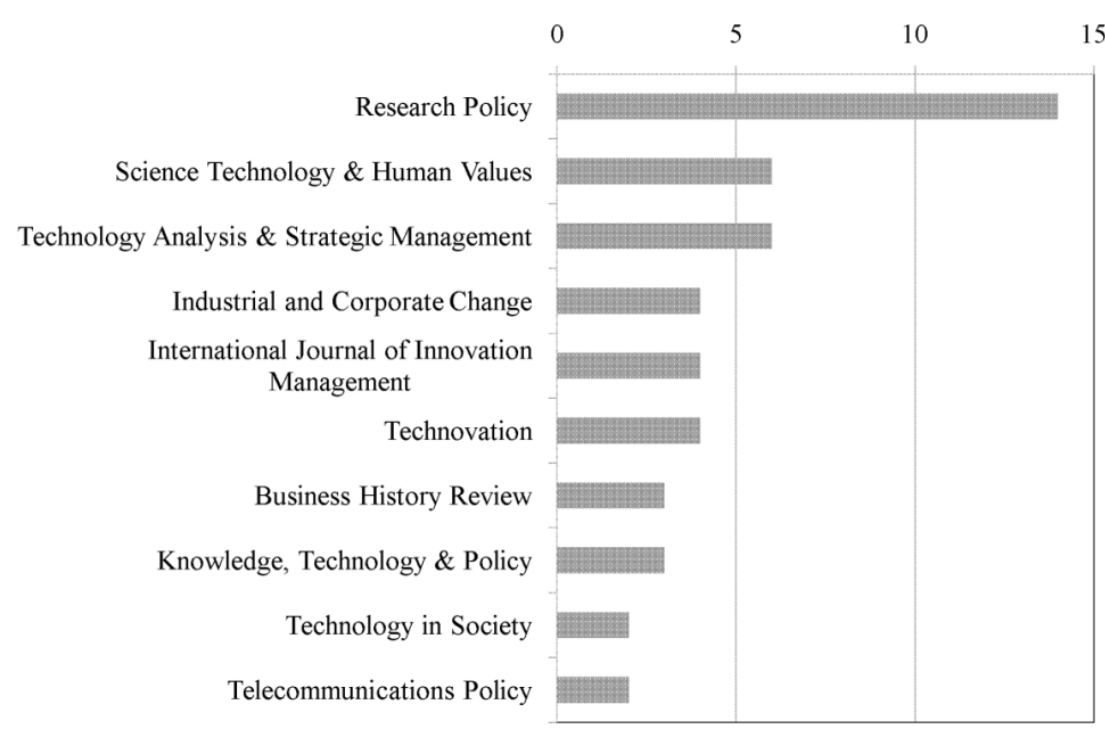

Figure 2. "Most frequently citing journals".

Research Policy accounts for 14 out of the 97 total citations, significantly more than any other journal. The journals Science, Technology \& Human Values, and Technology Analysis \& Strategic Management have both published six articles each and rate second in the overall frequency listing. It is additionally interesting to note that nine out of the total of 59 different journals (i.e. 15\%) which have cited the reverse salient concept over time have been policy oriented journals. This finding may indicate the utility of the concept in policy formulation or the call for policy changes, especially with respect to new technological systems that face performance limiting factors. Further, the change in the number of different journals which cite the concept over time is shown in Figure 3.

Since the first journal that cited the reverse salient concept in 1990, the number of journals which have published the concept has grown steadily over the timeframe of analysis. This finding confirms our second proposal and therefore indicates the broadening of the fields of research that have assigned value to the concept.

A second indicator of conceptual dissemination is found in the contexts, in other words the technological systems, which have been analyzed by scholars. Figure 4 
shows the frequency of citation of the reverse salient concept in the study of different research fields.

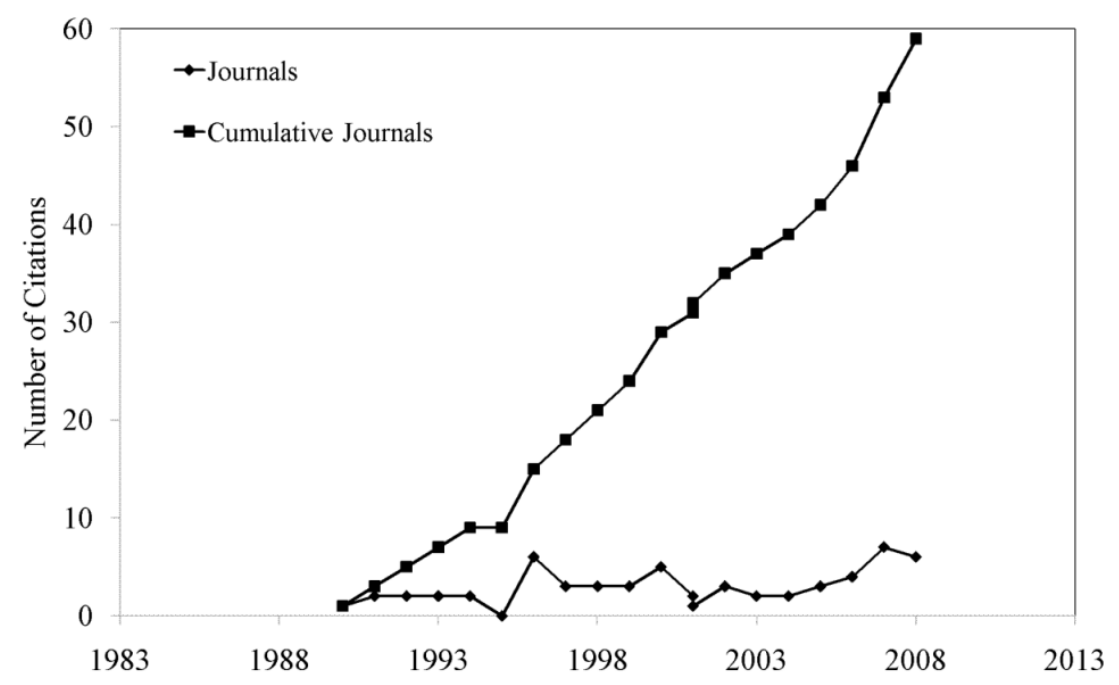

Figure 3. "Citations of the reverse salient concept in journals".

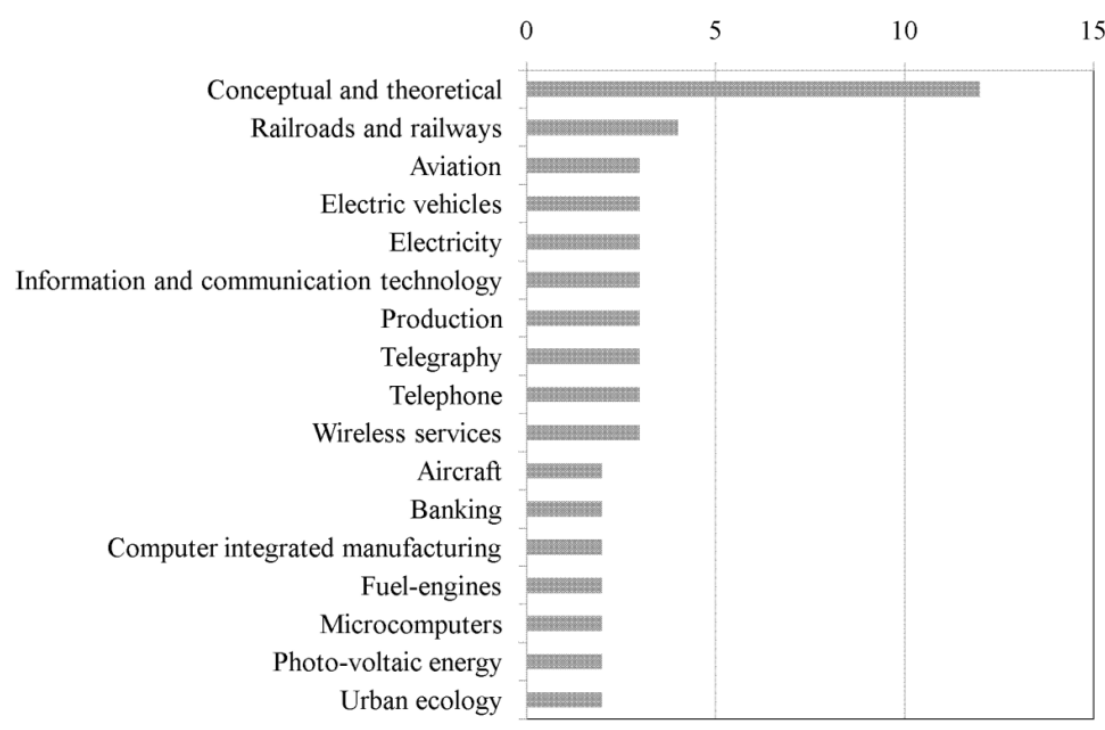

Figure 4. "Most frequently citing research fields (studied technological systems)".

Figure 4 illustrates that the most frequently citing articles are conceptual and theoretical (12 in all) in their nature and have not analyzed a particular technological system. The technological system which has been studied most frequently with respect to the reverse salient is the railroads and railways (four citations), followed by a number of other systems (three citations) including aviation, and information and communication technological systems. Interestingly, 
the research fields listed in Figure 4 are quite often either historical systems (e.g. telegraphy, railroads and railways) which have necessitated retrospective analysis of reverse salients, or new systems (e.g. photo-voltaic energy, electric vehicle) which require prospective analysis. The evolution of the number of research fields citing the reverse salient concept over time is shown in Figure 5.

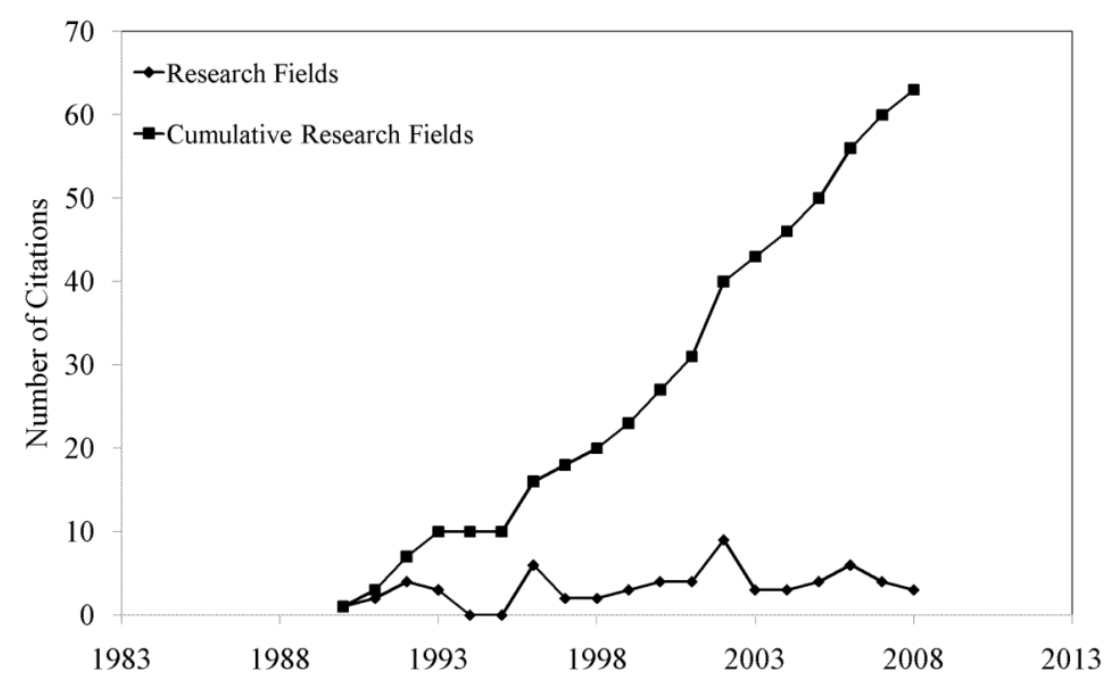

Figure 5. "Citations of the reverse salient concept in research fields".

Once again there appears to be a gradual increase in the total number of different research contexts which have incorporated the concept over time. This result is in line with our third proposal and again confirms our expectation of the pattern of concept dissemination in scientific research.

Finally, we compare the depth of conceptual application in citing articles over time in Figure 6.

Our study revealed 46 articles that had applied deep conceptual analysis in comparison to 51 articles which had employed shallow analysis. Figure 6 further indicates that the number of citations in both deep and shallow analysis has remained fairly similar across time. Nevertheless, there appears to be a persisting lag in the early period until 2001, where the deep analysis articles trail the shallow analysis articles in number of citations. This outcome is consistent with our fourth proposal which stipulates a period of ferment early after the concept's inception, due to limited knowledge of the concept and its potential. However, and as also indicated by Figure 6 , the deep analysis citations show increase at a later period, 
albeit short lived, overtaking the shallow citations in number. This result yet again supports our fourth proposal, although the strength of this support is arguable, considering in particular the most recent period where shallow analysis citations once again outnumber the deep analysis citations.

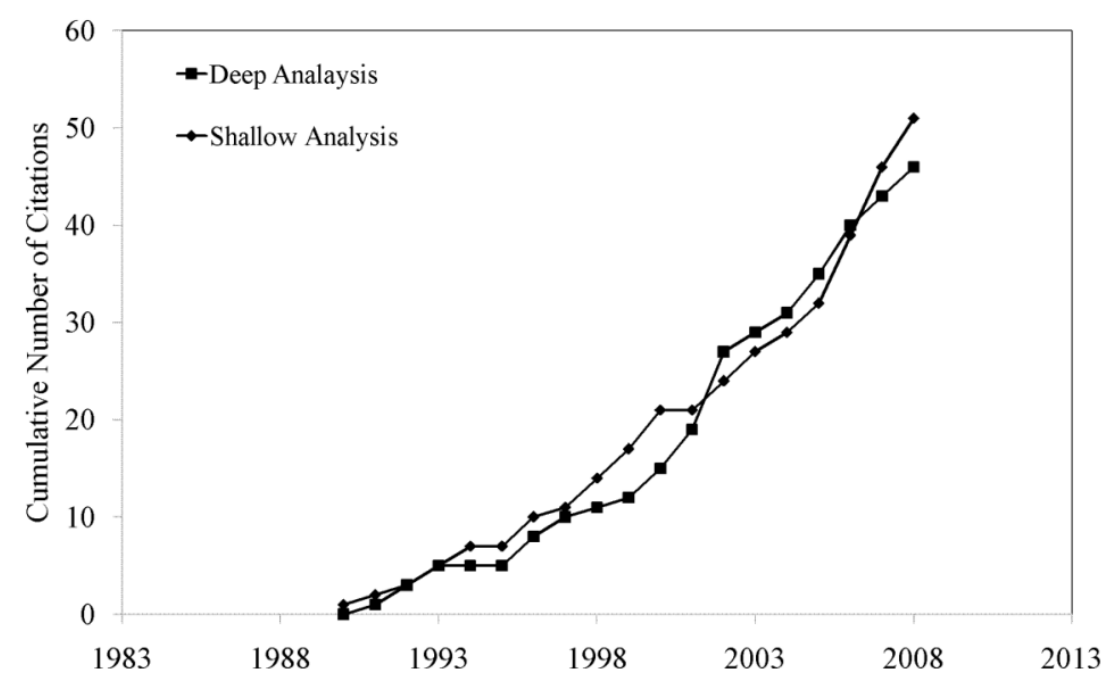

Figure 6. "Depth of conceptual application in citing articles".

\section{Discussion and conclusions}

The life span of a particular scientific work is an indication of the significance of that work in the literature. Seminal works or concepts are likely to receive high number of citations from the scientific community for many years, while works carrying less value are likely to experience short-lived and limited adoption after their inception. Cano and Lind (1991) have observed that seminal works can display pronounced increase in citations within the first four to seven years after being introduced, followed by a period of decrease in citations. While their study has focused on the medicine and biochemistry disciplines, their findings nevertheless offer some guideline as to the expected life of important scholarly contributions in other disciplines as well. Thus, using Cano and Lind's work as a frame of reference, we find that Hughes' reverse salient concept has continued to be perceived as valuable and has subsequently received a growing number of citations in the literature from its inception in 1983 until and including 2008. Furthermore, the dissemination pattern of the concept in periodicals as well as the number of studied contexts indicates similar increase. These results reaffirm once again the reverse salient concept's perceived value to scholars. These findings have 
important implications for the scientific community as well as the community of practitioners who select and employ conceptual tools in their analysis of technological systems, whereby the apparent scientific value of the concept increases its scope for adoption in future studies in academia and practice.

The reverse salient concept has been adopted and cited by scholars within a wide scope of research fields. For example, despite its initial application in 1983 within the context of the electric technological system, the reverse salient has been used in fields as far away as agricultural science, banking, urban systems, musical instrument technology, and government or public policy. The socio-technical characteristic of the concept affords flexibility, which has clearly been manipulated by scholars within literature at large. Not surprisingly, the concept has also continued to be used within a close radius of its origin, exemplified in particular by a number of articles covering the field of production and energy systems.

There are other interesting observations which can be made with respect to the research areas where the concept has been highly cited as well as areas where it has not been cited at all. Firstly, the reverse salient concept appears to be utilized in the analysis of alternative energy technological systems, which is presently a topic of high interest. A good example is Christiansen and Buen's (2002) article, which provides a comprehensive application of the reverse salient concept in assessing the case of photovoltaic and wave power development in Norway. We forecast an increase in the number of studies which apply the reverse salient concept in this and similar research fields, where the development of technological systems is often limited by both technical and social components of the system. In this case, the reverse salient provides a very fitting conceptual tool in the analysis of these systems.

Another general research discipline which cites the concept frequently is that of telecommunications, both in the modern and historical contexts. The articles dedicated to modern telecommunication cover topics such as wireless services and information and communication technology, which due to complex socio-technical systemic structures are predicted to continue publishing works citing the concept. It is also not surprising to observe the association of the reverse salient concept with the retrospective analyses of the telecommunication technological system. The telephone and telegraph technological systems have been analyzed in historical 
context, following Hughes' initial analysis of the closely related electric technological system's development.

Notwithstanding, there are relatively unexplored fields of research in which the reverse salient concept has potential to be incorporated - corresponding to research areas which have either cited the concept sparingly or not at all. One such arena is strategic management. The present study shows limited citation of the reverse salient concept inside strategic management circles. For instance, the Technology Analysis \& Strategic Management journal cites the reverse salient concept in only six out of the 97 articles considered in this study. Hence, we believe that the study of technological systems within the strategic management literature has potential in incorporating the reverse salient concept and has therefore not yet been exploited. Moreover, such application will require the increasing number of deep conceptual use, which currently accounts for approximately half of the cases studied.

Although some noteworthy conclusions have been drawn from the study presented here, some of the limitations of the study need to be brought forward to the reader's attention. Firstly, the citation list constructed from the research activity account only for the articles which appear in journals. Consequently, citations in books, monographs, working papers, and conference proceedings are not taken into account. This circumstance may have led to the exclusion of some sources where the reverse salient has been cited and therefore pinpoints one avenue of extending the present study. Notwithstanding, focusing on research within journals holds some merit in the sense that these publications are arguably more comprehensive than other modes of communication in scholarly discipline (Chandy $\&$ Williams, 1994). Journal articles therefore may provide a more suitable source of literature for content analysis as utilized in this paper, where focus is on researching the idiosyncrasies of a concept's use in addition to its citation alone. Our employed method of citation analysis has additionally received critique as an imperfect measure of research impact. This is because although some research may have impact or prove to be excellent scientific work it can still remain sparingly cited. Conversely, some research may receive high number of citations despite having less impact and overall value. Hence, while citation analysis remains widely used in bibliometric studies, its true indication of the impact of a seminal work or concept in the literature needs to be assessed with care. 
Another limitation of our study is that the initial pool of journals researched in this study has been constrained to those which have been within access. The inclusion of additional article databases in conducting this research, such as Google Scholar (see for example: Clarke, 2008), can enlarge the array of articles that are garnered. This however, remains a limitation of the present paper which can be address in future work. Additionally, future studies can assess the life cycle of the reverse salient concept by fitting curves (e.g. S-curve) on the attained cumulative citation graphs, which can illuminate the future citation behavior of the concept (Schwartz \& Fang, 2007). Other supplementing research, using citation and content analyses of related concepts such as technological imbalance (Rosenberg, 1976) and bottleneck can help ascertain how academic literature perceives the reverse salient concept on a relative scale.

In summary, it is proposed that literature holds two sets of potential concerning the use of the reverse salient concept which is found to be seminal in technology system analysis. Firstly, there lies an opportunity for a higher ratio of scholars to derive deeper conceptual utility in their work. Secondly, and related to the first, the application of the reverse salient concept remains poised to incorporate a more strategic approach to technological system analysis undertaken thus far in literature. We believe that the realizations of these potentials will benefit the literature concerned with the study of technological system in general and at the same time further develop the reverse salient concept.

\section{References}

Bijker, W. E., Hughes, T. P., \& Pinch, T. J. (Eds.). (1987). The social construction of technological systems. USA: The MIT Press.

Brown, L. D., \& Gardner, J. C. (1985). Applying citation analysis to evaluate the research contributions of accounting faculty and doctoral programs. The Accounting Review, 60(2), 262-267.

Callon, M. (1987). Society in the making: The study of technology as a tool for sociological analysis. In W. E. Bijker, T. P. Hughes \& T. P. Pinch (Eds.), The social construction of technological systems (pp. 83-106). USA: The MIT Press.

Cano, V., \& Lind, N. C. (1991). Citation life cycles of ten citation classics. Scientometrics, 22(2), 297-312. 
Chandy, P. R., \& Williams, T. G. E. (1994). The impact of journals and authors on international business research: A citational analysis JIBS articles. Journal of International Business Studies, 25(4), 715-728.

Christiansen, C. A., \& Buen, J. (2002). Managing environmental innovation in the energy sector: The case of photovoltaic and wave power development in Norway. International J ournal of Innovation Management, 6(3), 233-256.

Clarke, R. (2008). An exploratory study of information systems researcher impact. Communications of the Association for Information Systems, 22, 1-32.

Constant, E. W. (1987). The social locus of technological practice: Community, system, or organization? In W. E. Bijker, T. P. Hughes \& T. P. Pinch (Eds.), The social construction of technological systems (pp. 233-242). USA: The MIT Press.

den Hond, F. (1998). The 'similarity' and 'heterogeneity' theses in studying innovation: Evidence from the end-of-life vehicle case. Technology Analysis and Strategic Management, 10(4), 529-543.

Fransman, M. (2001). Analysing the evolution of industry. Economics of Innovation and New Technology, 10(2), 109-141.

Geels, F. W. (2006). Co-evolutionary and multi-level dynamics in transition: The transformation of aviation systems and the shift from propeller to turbojet (19301970). Technovation, 26(9), 999-1016.

Geyer, A., \& Davies, A. (2000). Managing project-system interfaces: Case studies of railway projects in restructured UK and German markets. Research Policy, 29(7), 991-1013.

Hargens, L. L. (2000). Using the literature: Reference networks, reference contexts, and the social structure of scholarship. American Sociological Review, 65(6), 846865.

Henderson, R. M., \& Clark, K. B. (1990). Architectural innovation: The reconfiguration of existing product technologies and the failure of established firms. Administrative Science Quarterly, 35(1), 9-30. 
Hobday, M. (1998). Product complexity, innovation and industrial organisation. Research Policy, 26, 689-710.

Hughes, T. P. (1983). Networks of power: Electrification in western society, 18801930. USA: The John Hopkins University Press.

Hughes, T. P. (1987). The evolution of large technological systems. In W. E. Bijker, T. P. Hughes \& T. P. Pinch (Eds.), The social construction of technological systems (pp. 51-82). USA: The MIT Press.

Kassarjian, H. H. (1977). Content analysis in consumer research. The Journal of Consumer Research, 4(1), 8-18.

Lariviere, V., Archambault, E., \& Gingras, Y. (2007). Long-term variations in the aging of scientific literature: From exponential growth to steady-state science (1900-2004). Journal of the American Society for Information Science and Technology, January, 288-296.

Ley, D. (2003). Forgetting postmodernism? recuperating a social history of local knowledge. Progress in Human Geography, 27(5), 537-560.

MacKenzie, D. (1987). Missile accuracy: A case study in the social processes of technological change. In W. E. Bijker, T. P. Hughes \& T. J. Pinch (Eds.), The social construction of technological systems (pp. 195-222). USA: The MIT Press.

Markard, J., \& Truffer, B. (2006). Innovation processes in large technical systems: Market liberalization as a driver for radical change? Reseach Policy, 35(5), 609625.

Mulder, K., \& Knot, M. (2001). PVC plastic: A history of systems development and entrenchment. Technology in Society, 23, 265-286.

Murmann, J. P., \& Frenken, K. (2006). Toward a systematic framework for research on dominant designs, technological innovations, and industrial change. Research Policy, 35, 925-952.

Prencipe, A. (2000). Breadth and depth of technological capabilities in CoPS: The case of the aircraft engine control system. Research Policy, 29, 895-911. 
Rosenberg, N. (1969). The direction of technological change: Inducement mechanisms and focusing devices. Economic Development and Cultural Change, 18, 1-24.

Rosenberg, N. (1976). Perspectives on technology. Cambridge: Cambridge University Press.

Rosenkopf, L., \& Nerkar, A. (1999). On the complexity of technological evolution: Exploring coevolution within and across hierarchical levels in optical disc technology. In J. A. C. Baum, \& B. McKelvey (Eds.), Variations in organization science (). USA: Sage Publications, Inc.

Schwartz, F. W., \& Fang, Y. C. (2007). Citation data analysis on hydrogeology. Journal of the American Society for Information Science and Technology, 58(4), 518-525.

Schwartz, F. W., \& Ibaraki, M. (2001). Hydrogeological research: Beginning of the end or end of the beginning? Ground Water, 39(4), 492-498.

Takeishi, A., \& Lee, K. (2005). Mobile music business in Japan and Korea: Copyright management institutions as a reverse salient. Journal of Strategic Information Systems, 14, 291-306.

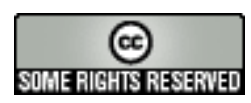

Article's contents are provided on a Attribution-Non Commercial 3.0 Creative commons license. Readers are allowed to copy, distribute and communicate article's contents, provided the author's and Journal of Industrial Engineering and Management's names are included. It must not be used for commercial purposes. To see the complete license contents, please visit http://creativecommons.org/licenses/by-nc/3.0/. 